THE SHEFEX FLIGHT EXPERIMENT ${ }^{(1)}$

\title{
- PATHFINDER EXPERIMENT FOR A SKY BASED TEST FACILITY -
}

\author{
J.M.A. Longo ${ }^{(2)}$, Th. Eggers \\ German Aerospace Center (DLR), Institute of Aerodynamics and Flow Technology, 38108 Braunschweig \\ J.Turner \\ TwIG, contracted by DLR, Mobile Rocket Base MORABA, 82234 Wessling \\ W. Jung, M. Hörschgen, A. Stamminger \\ DLR, Mobile Rocket Base MORABA, 82234 Wessling \\ A. Gülhan, F. Siebe, G. Requardt \\ DLR, Institute of Aerodynamics and Flow Technology, Wind Tunnel Division, 51147 Cologne \\ T. Laux, T. Reimer, H. Weihs \\ DLR, Institute of Structures and Design, 70503 Stuttgart
}

\section{ABSTRACT}

On Thursday, October 27th, 2005 the SHarp Edge Flight EXperiment SHEFEX has been successfully launched at the Andøya Rocket Range in northern Norway. The project, being performed under responsibility of the German Aerospace Center (DLR) flew on top of a two-stage solid propellant sounding rocket. One purpose of the experiment is the investigation of possible new shapes for future launcher or re-entry vehicles applying a shape with facetted surfaces and sharp edges and to enable the time accurate investigation of the flow effects and their structural answer during the hypersonic flight from $90 \mathrm{~km}$ down to an altitude of 20 $\mathrm{km}$. Additionally, the SHEFEX project is a starting point for a series of experiments which enable the acquisition of important knowledge in hypersonic free flight experimentation and which are an excellent test bed for new technological concepts. The present paper gives an overview about the philosophy and the layout of experiment and introduces preliminary outcomes of the post-flight analysis.

\section{INTRODUCTION}

Hypersonic systems are complex, difficult to design and expensive to build due to a lack of physical understanding on the involved flow regimes and a lack of data for design. Although in each decade considerable energy was spent designing new concepts and developing the technology base required supporting them, no aircraft capable of flying at Mach 10 or higher has been built and the current space transportation systems do not enable routine low-cost operations [1]. In order to improve the reliability of accessing space, e.g. problems related to vehicle servicing and refurbishing, must be greatly simplified and the time required for a design cycle has to be drastically reduced. The required technological developments are directly associated with technological progress of the three disciplines:

\footnotetext{
${ }^{1}$ An extended version of this study is simultaneously presented at the AIAA Aerospace Plane Conference, Australia, paper AIAA-2006-7921

${ }^{2}$ SHEFEX Project Manager, Jose.Longo@dlr.de
}

Aero(thermo)dynamics, Propulsion, Structures and Materials. A close interaction of these three disciplines, as well as the optimal use of all technical potentialities is necessary for a drastic reduction of design times, improved vehicle performance and operations. The strategic tool which one day will enable a dramatic reduction in the design and development time required for new vehicles is the computational or "virtual" vehicle design and qualification. But this approach is based on mathematical models which require verification and validation to increase their credibility. The improvement of the physical modelling requires good data, acquired in ground facilities and in flight, to be used for code validation and when necessary also code calibration. Ground based facilities, the major source of flow data, are very important because they allow a better understanding of the flow physics, but in-flight measurements are the only way to obtain data for prediction tool validation and calibration under real conditions (Fig. 1). Therefore, they are irreplaceable for physical modeling [2-3].
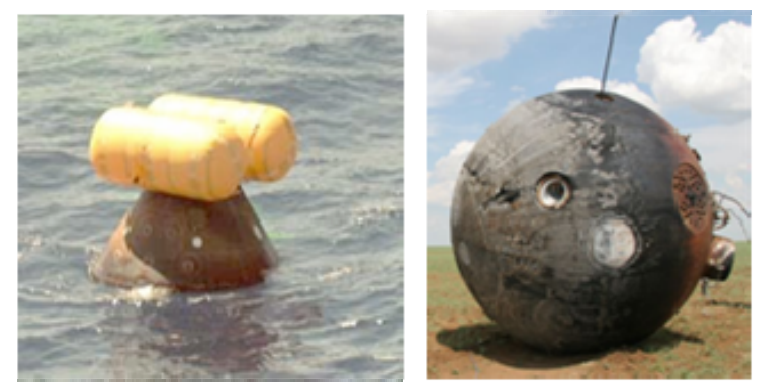

FIG. 1. ARD and FOTON shortly after landing (courtesy of ESA \& DLR)

In the last 25 years the achievement of progress in hypersonic physics has often been strongly coupled with new operational system developments instead of using less complex systems like sounding rockets as test bed for new technological concepts or simply for the gathering of flight data which are representative of the real hypersonic environments. Many X-programs, such as Hermes, the X-30, Sänger, X-33, X-34 and the 
X-38 have failed, in some cases shortly before the flight test that would have produced the badly needed data. Those failures occurred partially due to economical reasons and partially due to the technological challenges associated with the projects, the first cause being also closely related to the second one. In analogy to the ground based facilities, sounding rockets perhaps may be the way of access to a certain type of "free-flight facility", comparable in price also with today's so-called "industrial wind tunnels", but in any case, extremely less expensive and less risky than the so-called " $X$ vehicles". An example of hypersonic experiments on sounding rockets is the NASA Sub-Orbital Aerodynamic Reentry EXperiment SOAREX program [4] to test future flight vehicles. Another example of such strategy is the HyShot experiment of the University of Queensland [56]. At DLR this approach was adopted during the definition of the SHEFEX project as a pathfinder experiment for a series of hypersonic experiments that will also be flown on sounding rockets.

\section{DEFINITION OF THE EXPERIMENT}

The objective of the SHEFEX experiment was the investigation of a facetted Thermal Protection System (TPS) concept and the assessment of the potential of sharp edged configurations applying the three point strategy: numerical analysis - ground based facilities flight experiment. The motivation is neither to perform a re-entry experiment nor to fly at the thermal boundary of modern high temperature materials but to prove in flight that the temperature peaks at the edges of the ceramiccomposite panels are lower than those predicted based on a radiation equilibrium hypothesis.

\subsection{Aerodynamic Motivation and Layout}

From the aerodynamic point of view, the design of a hypersonic vehicle is influenced by finding a compromise between a vehicle being sharp enough to obtain acceptable aerodynamic and propulsion efficiency and blunt enough to reduce the aerodynamic heating. Indeed, sharp edged configurations offer more gradual kinetic energy conversion and flat heat flux time profile along the upper part of the re-entry trajectory avoiding high peaks in the energy dissipation and heat flux rate; controllability of the vehicle along the entire flight path; low pressure forces and decelerations; low landing speed; a very large landing footprint due to the long reentry duration; no blackout in radio-communications and low angles of attack for efficient control surfaces operations. Therefore, during the 1990s, the development of ceramic composite and ultra high temperature materials for TPS applications led to a renewed interest in sharp edged configurations such as the waverider concept DLR F8 [7], the DLR-ONERA project JAPHAR [8] or the lifting body concept HL-20 and the SHARP project, both from NASA [9], [10]. Driven by the facetted concept, two main criteria have been used to define the aerodynamic shape of the SHEFEX forebody. It should have as many as possible facetted panels and it should represent as many as possible configuration details of space vehicles, like concave and convex chamfers and a sharp unswept leading edge (Fig. 2).

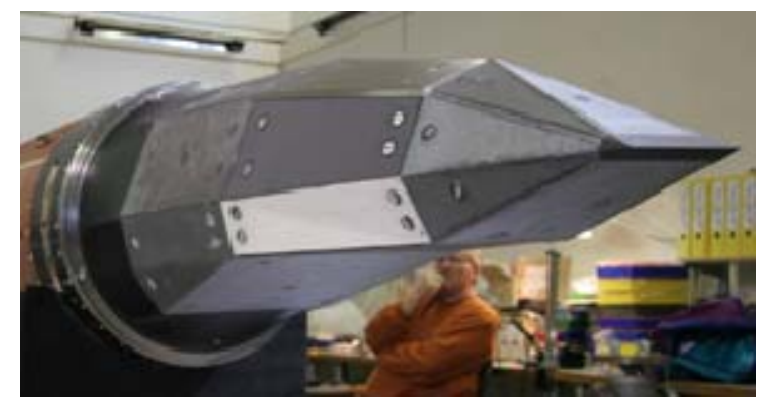

FIG. 2. The SHEFEX Experiment

As summarized in [11] and [12] the aerodynamic layout has been performed based on Euler- and Navier-Stokes calculation, applying the DLR TAU code [13] on unstructured grids. The main purpose of the aerothermodynamic analysis of the SHEFEX forebody is the assessment of the expected heat loads and a first analysis of the flow field. The obtained surface values of pressure, temperature and heat flux have been basis for the choice of the flight instrumentation, the positioning of the sensors and for the layout of structure and TPS (Fig. 3).

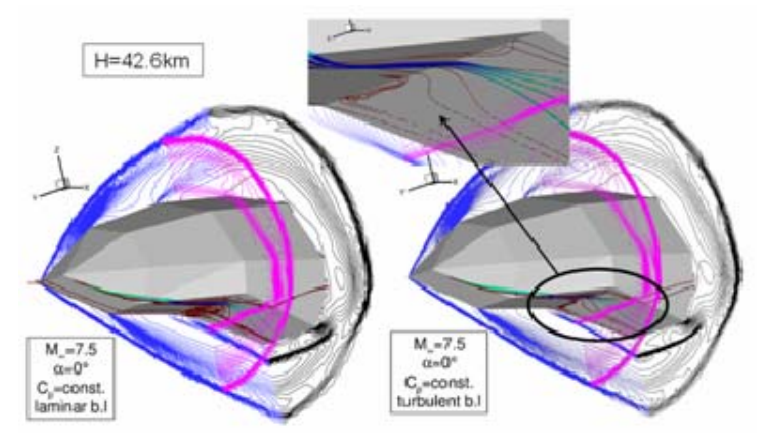

FIG. 3. Boundary layer impacts on surface flow field

The launcher is a two stage solid propellant sounding rocket consisting of a Brazilian S30 motor as first stage and an Improved Orion motor as second stage. Between the facetted forebody and the second stage are two cylindrical modules which housed the recovery system, the main electronics, the data acquisition devices, the power supply and the cold gas ACS. During ascent the facetted forebody is protected by an ogive nose cone. (Fig. 4).

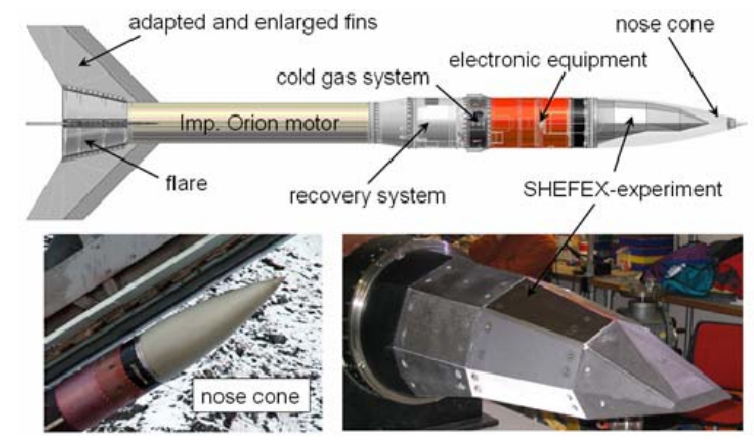

FIG. 4. SHEFEX re-entry configuration 
The aerodynamic layout of the re-entry configuration is defined applying Euler calculations. At first sight, the technical requirements for the performance of the SHEFEX mission appeared relatively straightforward. But the behavior of the re-entry configuration is dominated by the lift of the asymmetric payload which drastically reduced the static margin. The main challenge of the design has been the definition of a configuration being stable with respect to the longitudinal as well as the lateral motion. In order to reduce the drag as well as the structural loads, the angle of attack has to be as small as possible. Complicating the conditions for the layout is the fact that the fins of the second stage shall be uncanted and that the adaptation of the overall center of gravity for the re-entry configuration is very demanding due to the high mass of the burnt-out second stage motor. A first analysis of several fin arrangements with a view to the static margin pointed out that only a four fin configuration with two horizontal and two vertical fins would enable the definition of a realistic reentry configuration. Additionally, the fins had to be designed as large as possible however the fin size is restricted by the ascent stability. An additional flare in the back of the vehicle also is introduced to increase the static margin. The resulting layout of the vehicle exhibited enlarged fins for the second stage adapted to the new flare. This change together with its uncanted arrangement, which is required to prevent spin during descent, forced also a redesign of the first stage fins and the corresponding tailcan, since the modifications of the re-entry vehicle reduced the vehicle stability during ascent. Therefore, also for the first stage a new tailcan and fins has been developed and the SHEFEX team ended up with a completely redesigned launcher (Fig. 5).

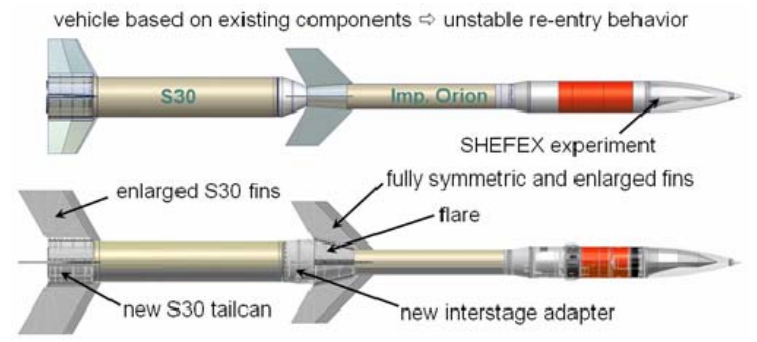

FIG. 5. Layout of the SHEFEX launcher

\subsection{Structure and TPS}

The SHEFEX structure consisted of a primary aluminum structure and the thermal protection panels [14]. All in-flight measurement techniques are integrated into the thermal protection system (TPS) and housed in the primary structure. This strategy allowed test panels of different materials and/or surface coatings; direct access to the primary structure and the easy replacement of type and/or distribution of sensors. That is, each panel could be used as a self contained experimental platform. The basic substructure consisted of an aluminum frame created by stiff booms and spars. The free space is closed by flat aluminum panels, which are also used for the mounting of the TPS panels and the instrumentation. Inside the frame, some measurement equipments are integrated. These items are a thermocouple connection and compensation box, pressure transducers and a pyrometer (Fig. 6).

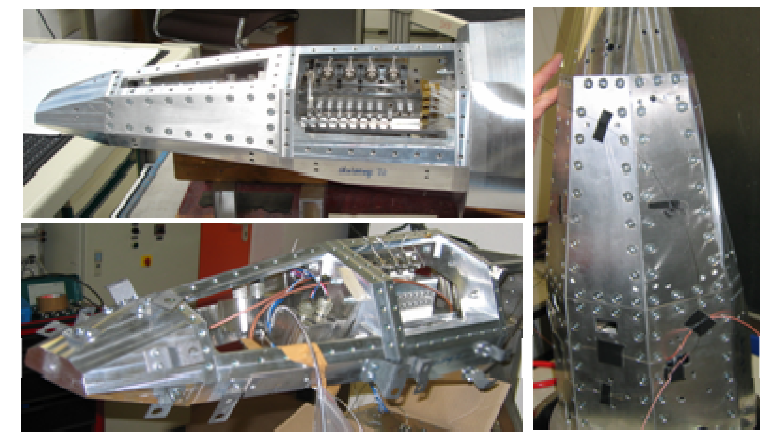

FIG. 6. SHEFEX primary aluminum structure

The main thermal protection system has been designed using a segmented concept with CMC panels manufactured with DLR's liquid silicon infiltration process, being developed within former ESA programs. The main element of the concept is a fiber ceramic cover plate, supported in all directions by a so called central post and flexible stand offs at the corners. Thus, the thermal expansion was not suppressed. Beneath the cover plate a lightweight fibrous ceramic insulation mat was inserted. A key element of this TPS concept is a ceramic fastener, used for the connection of the panel to the CMC stand off and central post. In fact, all facets of the second and third segment are created by a single CMC panel (Fig. 7).
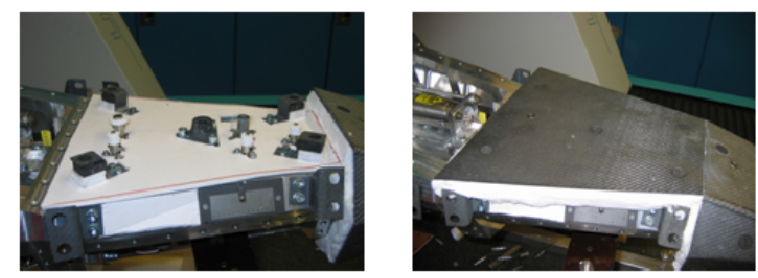

FIG. 7. WHIPOX insulation concept

Another key technology is the design of the sharp leading edge, which shall be exposed to a very high heat flux during re-entry. The DLR's C/C-SiC material, which has demonstrated very moderate erosion behavior at extreme heat loads in the past, has been used for the flight of the SHEFEX vehicle. A further new element of the TPS is the seal design at the interfaces between the panels. Since the shape of SHEFEX includes a certain number of different angles at the interface chamfers, a lot of different single rigid seal parts would be necessary. This causes a high manufacturing effort because of adopting the rigid seal contour close to the different panel shapes. During recent years an oxide ceramic based CMC material was developed at DLR. The so called WHIPOX material, which mainly consists of alumina and silica, can be used as an oxidation stable alternative to carbon based CMC's. However, temperature stability is limited and a special coating is necessary to improve emissivity and catalytic behavior. Nevertheless, this material provides a flexible intermediate state during the manufacturing process. Thus, it is possible to shape a component (in case of SHEFEX, the rigid seal) during assembly. Hardening would occur during re-entry. Using this property, it is possible to shape and cut all required seal components from one uniform WHIPOX tape (Fig. 8). A typical seal interface 
has been tested in the arc heated facility LBK of DLR Cologne and handling procedure, seal performance and temperature stability have been demonstrated successfully.

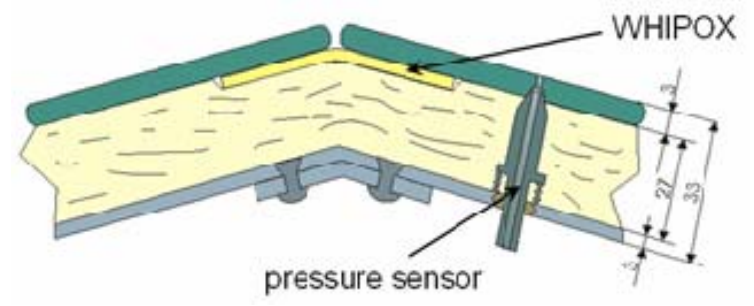

FIG. 8. WHIPOX sealing concept

In addition some passenger TPS experiments have been integrated into the SHEFEX vehicle. Adjacent to the C/C-SiC bottom plate, a cured WHIPOX panel was integrated on each side of the vehicle. The remaining facets of this segment are closed by four different TPS samples provided by EADS; two ceramic based and two metallic based concepts. Both metallic TPS experiments have been delivered by Plansee, Austria. Finally, an additional experiment from MT Aerospace has been integrated within the third segment.

\subsection{Instrumentation}

The definition of the type and location of the sensors has been carried out based on the data of a detailed numerical analysis (Fig. 9). Thermocouples, heat flux sensors and pressure transducers are used to measure the aerothermal heating on the panels and inside the sub-structure. The maximum number of sensors i.e. analogue channels, are defined by the onboard data handling and telemetry systems. Finally the following instrumentation has been used: 40 sheathed thermocouples of type $\mathrm{K}$ (sheath diameter $0.5 \mathrm{~mm}$ ), 2 sheathed thermocouples of type $S$ (sheath diameter 1.0 $\mathrm{mm}), 3$ Platinum resistance thermometers Pt 100, 5 heatflux sensors, 1 pyrometer and 8 pressure transducers. The mechanical interfaces have been designed and manufactured taking into account the demand for an adequate insensitivity to acceleration and upstream movement of the center of gravity for aerodynamic stability. The same has been done for the layout of the links from the measuring sites to the sensor inputs.

To measure the absolute temperatures with thermocouples a passive temperature reference consisting of a thermally slow-acting copper-block as "cold" junction has been used. The actual temperature of this temperature reference has been measured using high accuracy resistance thermometers (pt100) and has been transmitted via one of the telemetry channels for ground based compensation. To boost the low output voltages of the thermocouples to the input level of the ADconverter, 42 analog amplifier modules - 14 grouped on each of three identically designed PCBs - have been installed into the electronic box. The heatflux sensors (five units) are made of two components: a temperature sensitive resis $\neg$ tor (RTS) and a cascade of special thermocouples (HFS). For both of them special amplifier modules have been built: a differential amplifier for the thermocouple-cascade and a single ended amplifier for the RTS, taking into account the very low permissible feeding current. These modules have been grouped on a separate Heatflux-PCB, also installed into the electronic box. The bandwidth for the complete analogue signal processing was limited to $100 \mathrm{~Hz}$ by lowpass filters. The pressure sensors have been so called "active" ones, i.e. each of them is equipped with its own strain gauge feeding and amplifier. The pyrometer has been equipped in a similar way. To protect the ADC inputs, separating buffer amplifiers were provided for the active sensors.

To read and process the digital data of the payload, the data are transferred by hard line (for testing purposes in the laboratory or during the launch count down) and through telemetry in the course of flight. Aself developed software allows simultaneous saving of the raw data stream of the telemetry or of the hardline and provides a quick-look analysis involving preliminary calibrations. This software was used during all on ground testing and the final mission. It also ensured a constant health monitoring of the sensors during preparation and the mission. A detailed description of the instrumentation of the facetted payload is given in [15].
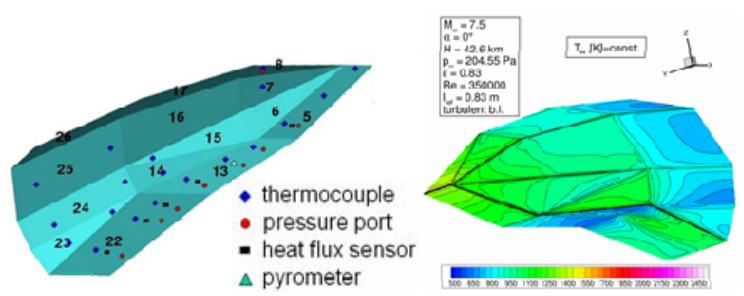

FIG. 9. Definition of the sensor position

\section{MISION}

The mission is schematically sketched in Fig. 10. The first stage burns out after $28 \mathrm{sec}$ and separates from the rest of the launcher at an altitude of $17 \mathrm{~km}$. The second stage burns out after $56 \mathrm{sec}$ at an altitude of 65 $\mathrm{km}$. Since the facetted body has no control devices the second stage remains attached to the facetted body until the end of the experiment to provide flight stability through its fins. At $90 \mathrm{~km}$, the nose cone separated and a cold gas ACS initiated the pointing maneuver. The apogee was reached at approx. $240 \mathrm{~km}$. No yo-yo despinning maneuver is necessary since the ACS eliminated any remaining spin. The SHEFEX experiment started in the descent at an altitude of $90 \mathrm{~km}$ and continued with an almost constant flight Mach number of about 6.0 down to an altitude of $20 \mathrm{~km}$. The selected flight path fits rather well inside the operational range of the high enthalpy ground facilities of DLR, allowing numerical and ground base data correlations with flight measurements. From the mission point of view, the challenge of SHEFEX is the definition of a re-entry configuration which has to fly aerodynamically stable without control surfaces and as originally planned without spin. 

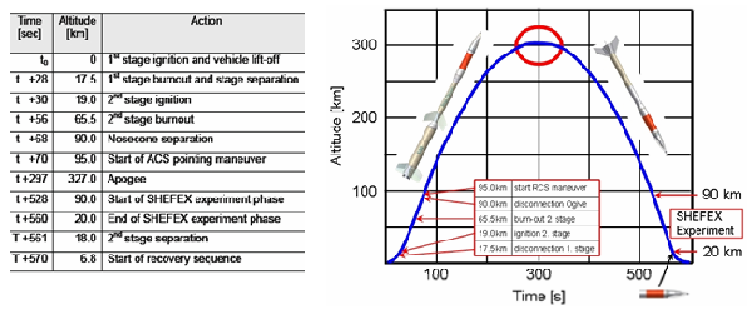

FIG. 10. Nominal SHEFEX mission (schematic)

An experiment time of approx. $45 \mathrm{sec}$ is available for measurements in the hypersonic regime. After separation of the 2nd stage the payload module with the SHEFEX experiment changes to an unstable flight and decelerates in an almost flat spin mode to a recovery velocity of approximately $250 \mathrm{~m} / \mathrm{s}$. At an altitude of $4 \mathrm{~km}$ the recovery sequence should have started with the deployment of a two-stage parachute system to provide a final velocity of approximately $15 \mathrm{~m} / \mathrm{s}$ and safe landing. As described in more depth in [16] the main sensor for the attitude control is a stabilised inertial platform. It provides accurate attitude and orientation information. The platform also provides the attitude control signal for the automatic cold gas ACS system. A main microcontroller performs the data acquisition from the platform, the experiment electronics, GPS receiver, ignition and recovery system and the general housekeeping data. The controller also assembles the various data packets in a PCM frame for telemetry and receives the telecommands via dual redundant receivers for correction of maneuvers or selective enabling of lateral and roll axes control. The baseline control strategy is a predefined maneuver according to the predicted nominal trajectory. In the event of a trajectory significantly differing from the nominal, the calculation of the flight vector is done from real-time radar data and the transmission of the maneuver correction is then uploaded via telecommand.

\section{SELECTED RESULTS}

SHEFEX has been launched on 27th October 2005 at 13:45 UTC. During flight the vehicle reached an apogee of $211 \mathrm{~km}$ which is $35 \mathrm{~km}$ lower than the expected altitude. The total flight time has been 550 seconds comprising 45 seconds of experimental time for the atmospheric re-entry between $90 \mathrm{~km}$ and $14 \mathrm{~km}$. The originally planned altitude of approx. $246 \mathrm{~km}$ could not be reached because the spin balancing tests required the mounting of an additional $14 \mathrm{~kg}$ of ballast. The flight velocities during the atmospheric decent varied in the region of $1700 \mathrm{~m} / \mathrm{sec}$. The maximum velocity of 1857 $\mathrm{m} / \mathrm{s}$ during re-entry has been measured at an altitude of $28.3 \mathrm{~km}$. The Mach number is relatively constant at a value of approx. 5.6 from $100 \mathrm{~km}$ down to $50 \mathrm{~km}$. Then the Mach number increases up to its maximum value 6.2 at $26 \mathrm{~km}$. From $100 \mathrm{~km}$ down to the payload separation it took only a time of around 52 seconds. When passing $25 \mathrm{~km}$ of altitude the onboard camera shows thermal degradation of the fin leading edges resulting in a sine shape deformation and glowing (Fig.11).
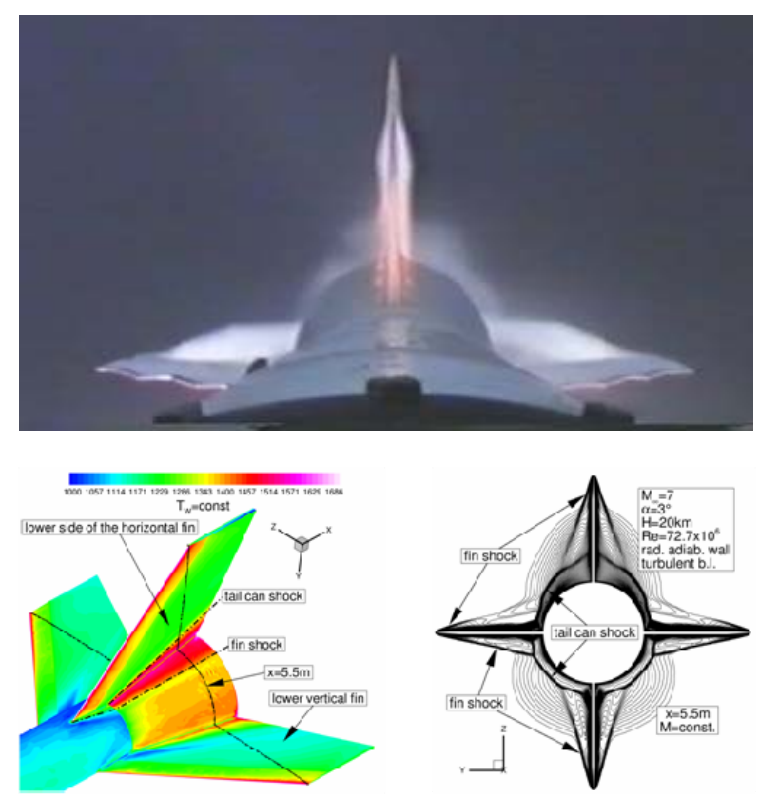

FIG. 11. Vehicle during re-entry and CFD predictions

At $14 \mathrm{~km}$ of altitude the motor separation has been initiated nominally by barometric switches, but unfortunately, also the recovery sequence has been activated too. As quick look data showed that the recovery system could not produce a soft landing and flotation in the water, the recovery vessel has been recalled.

From an analysis of the data received from the experiment and service systems it can be said that all major experiment aims have been achieved proving that the novel TPS system worked according to the expectations. In the light of the enormous scientific data, the failure to recover the payload has only an insignificant effect on the success of the SHEFEX mission. Based on the detailed analyses of the sensor data and the exact evaluation of the subsystem data like e.g. those from housekeeping sensors, DMARS, Radar, GPS and the onboard cameras a direct comparison of the flight data with numerical post flight studies is possible (Fig. 12).
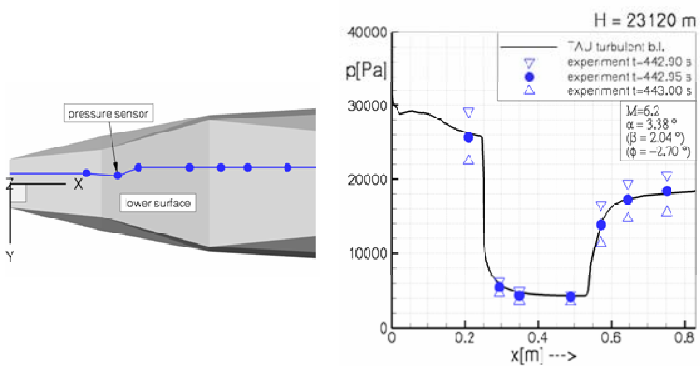

FIG. 12. Comparison of CFD and flight data [17]

\section{ONGOING ACTIVITIES}

The post flight analysis will deepen the understanding of the located uncertainties and in a careful step by step approach also the measured temperatures and heat flux rates will be taken into account once the reconstruction of the measured pressures is fully understood. 
Currently, it has to be pointed out that the pressure analysis is more complex than expected although the surface pressure is much less sensitive to predict than surface temperatures or heat flux rates. The next step of the post flight activities is the construction of a wind tunnel model for the High Enthalpy Shock Tunnel, HEG, at the DLR Göttingen. The model (Fig. 13) which currently is in fabrication is instrumented with 86 thermocouples and 66 pressure sensors. It is going to enable numerical and ground based data correlation with the flight measurement for a flight condition in approx. 19 $\mathrm{km}$. A more medium-term activity is a numerical coupling of flight mechanics and CFD which enables a direct prediction of the expected flight path of re-entry vehicles. Here the very detailed platform data are an excellent possibility for the validation of the acquired coupling approach.

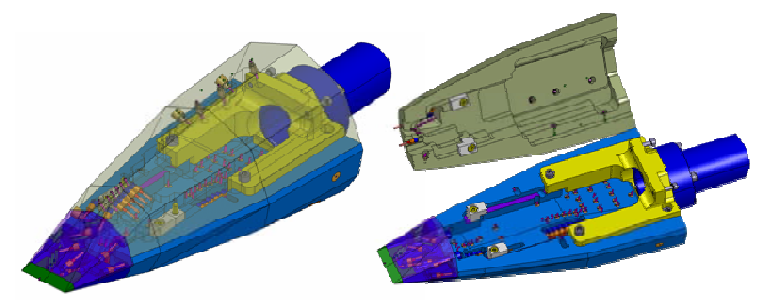

FIG. 13. CAD drawings of SHEFEX wind tunnel model

\section{FINAL REMARKS}

The given paper summarised the overall philosophy and the background of the hypersonic flight experiment SHEFEX of the German Aerospace Center, DLR. It gave a detailed overview about the SHEFEX flight campaign introducing the approaches of all involved disciplines, i.e. mission, system and launcher, aerodynamics, aerothermodynamics and in-flight measurement techniques as well as structure and thermal protection. Additionally, the paper highlighted the main outcomes and drawbacks of the SHEFEX flight, which are more deeply discussed in additional technical papers. The evaluation of the flight data points out that relatively straightforward experiment like SHEFEX and their enormous amount of scientific data emphasize, that free flight experiments are an essential source to acquire knowledge in the physics of hypersonic flight and the development of more complex vehicles. The application of this knowledge is a huge source of motivation and encouraged DLR to implement a step by step approach towards a fully controlled re-entry vehicle. Therefore the goal of the currently defined SHEFEX II experiment, planned to be launched in spring 2009, is a fully controlled and stabilized flight of a facetted re-entry configuration like SHEFEX with a symmetric facetted forebody at Mach numbers between $10<\mathrm{M}<12$.

\section{REFERENCES}

[1] Bertin, J.J., Cummings, R.M., Fifty years of hypersonic: where we've been, where we're going, Progress in Aerospace Sciences, 39 (2003) 511-536.

[2] Longo, J.M.A., Aerothermodynamics - A critical review at DLR, Aerosp. Sci. Technol. 7 (2003) 429-438.

[3] Longo, J.M.A., Modelling of Hypersonic Flow Phenomena, VKI-RTO Lecture Series "Critical Technolo- gies for Hypersonic Vehicle Development Technology", VKI, Belgium, 2004.

[4] AW\&ST, Waverider Results, Aviation Week \& Space Technology, November 10, 2003, pp. 17.

[5] University of Queensland, A description of the HyShot experiment, Web pages University of Queensland, www.uq.edu.au.

[6] Gardner, A.D.; Hannemann, K.; Steelant, J.; Paull, A., Ground Testing of the HyShot Supersonic Combustion Flight Experiment in HEG and Comparison with Flight Data, AIAA-Paper 2004-3345, 2004.

[7] Strohmeyer, D.; Eggers, Th.; Haupt, M.: Waverider Aerodynamics and Preliminary Design for Two-Stageto-Orbit Missions, Part 1, Journal of Spacecraft and Rockets, Vol. 35, No. 4, July-August 1998, pp. 450-458. [8] Eggers, Th.; Novelli, Ph.; Haupt, M.: Design Studies of the JAPHAR Experimental Vehi-cle for Dual Mode Ramjet Demonstration, AIAA Paper 2001-1921, 2001.

[9] Reuther, J. et al.: A Reusable Space Vehicle Design Study Exploring Sharp Leading Edges, AIAA Paper 2001-2884, 2001.

[10] Arnold, J.; Johnson, S.; Wercinski, P.:SHARP: NASA's Research and Development Activities in UltraHigh Temperature Ceramic Nose Caps and Leading Edges for Future Space Transporatation Vehicles, Proceedings of the 52nd International Astronautical Congress, IAF paper 01-V.5.02, France, October 2001. [11] Eggers, Th., Longo, J.M.A., Hörschgen, M., Stamminger, A.: The Hypersonic Flight Experiment SHEFEX, AIAA Paper 2005-3294, 2005.

[12] Eggers, Th., Stamminger, A., Hörschgen, M, Turner, J.: The Hypersonic Experiment SHEFEX Aerothermodynamic Layout, Vehicle Development and First Flight Results, Proceedings of 6th International Symposium on Launcher Technologies 'Flight Environment Control for Future and Operational Launchers', Munich, Germany, November 2005.

[13] Mack, A.; Hannemann, V., Validation of the Unstructured DLR-TAU-Code for Hypersonic Flows, AIAA Paper 2002-3111, 2002.

[14] Weihs, H., Reimer, Th., Laux, T., Mechanical Architecture and Status of the Flight Unit of the Sharp Edge Flight Experiment SHEFEX, Proceedings of the 55th International Astronautical Congress, Vancouver, Canada, 2004.

[15] Gülhan, A.; Requardt, G.; Siebe, F.; Koch, U.; Esser, B.: Instrumentation and In-Flight Data of the SHEFEX Flight Experiment, Proceedings of the 1st International ARA Days on Atmospheric Re-Entry Systems, Missions and Vehicles, Arcachon, France, June 2006.

[16] Turner, J.; Hörschgen, M.; Turner, P.; Ettl, J.; Jung, W.; Stamminger, A.: SHEFEX - The Vehicle And Subsystems for a Hypersonic Re-Entry Flight Experiment, 17th ESA Symposium on European Rocket and Balloon Programmes and Related Research, Sandefjord, Norway, June 2005.

[17] Turner, J.; Hörschgen, M.; Stamminger, A.; Turner, P.: SHEFEX Hypersonic Reentry Flight Experiment, Vehicle and Subsystem Design, Flight Performance and Prospects, AIAA Paper 2006-8115, 2006.

[18] Eggers, Th.; Barth, T.: Aerodynamic Analysis of the DLR Flight Experiment SHEFEX, AIAA Paper 20068027, 2006. 